\title{
Hydrometeorological monitoring for water balance determination at plot scale
}

\author{
A. Sommella, ${ }^{1}$ Mario Palladino, ${ }^{1}$ A. Comegna, ${ }^{2}$ A. Coppola ${ }^{3}$ \\ ${ }^{1}$ Division of Water Resources Management, University of Naples "Federico II", Italy; ${ }^{2}$ School of \\ Agricultural Forestry Food and Environmental Sciences (SAFE), University of Basilicata, Potenza, \\ Italy
}

\begin{abstract}
To provide adequate answers to recurring problems in land and water resources management, nowadays the use of physically based models is widespread. The reliability of the responses of this kind of model is closely linked to the precision with which model parameters are determined, such as soil hydraulic characteristics and meteorological variables. In this work, we set up a complete station for the monitoring of both meteorological and soil variables, as well as a soil hydraulic characterization. The experimental site shows vertic characteristics and is in Guardia Perticara, Basilicata region, Italy.
\end{abstract}

\section{Introduction}

The water balance of soil-plant-atmosphere system can be studied at different scales. The study at a smaller scale is useful if the goal is to define the basic laws concerning the physical phenomena occurring in the soil and the physiological processes occurring into plants; the medium scale when interested in the control of hydrological parcels of land cultivated mainly for agricultural purposes; bigger scale is most appropriate for comprehensive evaluations that affect entire river basins.

Medium scale approach is based on the definition of water balance, represented by an expression which summarizes the water cycle accomplished through fluxes of water in the soil and represented by the following relationship:

$\mathrm{N}+\mathrm{I}=\mathrm{ET}+\mathrm{Pr} \pm \mathrm{D}$

where the symbols, for a given period of time, are: $\mathrm{N}=$ natural incomes of water; I = irrigation amount; ET represents evapotranspiration fluxes over the period, $\mathrm{Pr}=$ loss of water to deep percolation and runoff; D $=$ variations in water content of soil profile.

Correspondence: A. Comegna, School of Agricultural Forestry Food and Environmental Sciences (SAFE), University of Basilicata, Potenza, Italy. E-mail: alessandro.comegna@unibas.it).

Key words: soil hydraulic characterization, soil water balance, TDR. (C) Copyright A. Sommella et al., 2013

Licensee PAGEPress, Italy

Journal of Agricultural Engineering 2013; XLIV(s2):e19

doi:10.4081/jae.2013.s2.e19

This article is distributed under the terms of the Creative Commons Attribution Noncommercial License (by-nc 3.0) which permits any noncommercial use, distribution, and reproduction in any medium, provided the original author(s) and source are credited.
In recent years it became common to physically based models to provide adequate answers to recurrent problems of management of land and water resources (Hillel, 1980; Santini 1992; Simunek et al., 1998). The reliability of these models is closely linked to the degree of accuracy with which the parameters are determined, such as soil hydraulic properties and meteorological variables.

To this end, on two plots of an experimental field site in Guardia Perticara (Potenza, Italy), was set up a complete instrumentation for the detection of these parameters.

The processes of transpiration, evaporation, infiltration and formation of runoff are largely determined by the content and the energy state of water in the unsaturated zone of the soil. Knowledge of the evolution in time and space of these quantities is a prerequisite for the identification of mass exchanges between the different components of the soil-plant-atmosphere through which compute comprehensive and reliable water balances.

Although physically based mathematical models allow very detailed descriptions of water transfer processes, their degree of accuracy in terms of predicting accuracy is however conditioned by the large number of input parameters involved. Because these parameters are used also in the process of validation of the models, it is necessary for them to be observed through accurate evaluation that require a large number of observations, given the inherent spatial-temporal variability of the porous media in the study.

The burden of such determinations in the open field has necessitated the development of measuring devices and automatic acquisition system, which also require sophisticated instrumentation and specifically developed control software.

This study provides details of the experimental installation characterized by a high degree of automation and the detailed space-time acquisitions of the variables in the study. As such, it allows us to come to a complete hydrological balance, whose reliability is verified through a simulation of water exchange processes in the soil-atmosphere, conducted using only input parameters independently determined. In the simulation the crop has been neglected, because during the period under investigation, the soil is without vegetation.

\section{Materials and methods}

The experimental area falls within the Agri basin, sub-basin of the Sauro, in Guardia Perticara, Potenza (Italy), at an altitude of $720 \mathrm{~m}$ above sea level, with an average annual temperature of $12{ }^{\circ} \mathrm{C}$ and an average annual precipitation of about $790 \mathrm{~mm}$.

The area is located on a geological formation defined as polychrome shale claystones and, in the superficial part, affected by landslides. The soil type falls within the "vertic ustorthens", according to the classification USDA. Other important properties are represented by a low permeability and vertic characters from the top of the clay soil, well evi- 
dent during the summer because of the cracks. The soil profile shows the presence of an Ap horizon, which extends between $0 \mathrm{~cm}$ and $30 \mathrm{~cm}$ and a second horizon Cca, which develops from $30 \mathrm{~cm}$ up to more than $100 \mathrm{~cm}$. The soil was characterized hydraulically through undisturbed soil sampling at three different depths in ten stations. In the laboratory, retention and hydraulic conductivity curves were determined (Sommella and Gaetani, 1990; Santini et al., 1995). For each depth investigated the average curves characteristics showed no significant differences between the different location (Figure 1). The field was divided into 16 plots, arranged in subplots of rectangular shape with a width of $15 \mathrm{~m}$ and a length equal to $40 \mathrm{~m}$, the average slope of each plot according to the longest side is equal to $14 \%$ while the transverse slope is virtually zero (Figure 2).

The 8 downstream plots have been hydraulically isolated from the others in order to precisely define the boundary conditions. For the rainfall measures three pluviographs were installed. To measure the parcels outflows were prepared three channels of $5 \mathrm{~m}$ length, made of sheet metal, immediately downstream of each parcel parallel to the shorter side. For each plot, the water runoff intercepted by the collecting channels were connected through PVC pipes with a diameter of 140 $\mathrm{mm}$ to a channel of supply; outflows were finally collected in a tank with a volume of $1 \mathrm{~m}^{3}$. For direct estimation of the volumes of groundwater a drainage system has been buried in the first soil horizon. With reference to the investigation in this study, the tests involved two of the eight parcels of the experimental field on which, in addition to the instrumentation described above, have been installed for each plot 6 access tubes for the use of the neutron probe and TDR probes, for water content monitoring, as well as tensiometers, for water potential measurements. For a complete and accurate data acquisition a centralized system was installed, which allowed the contemporary taking of the measures. The system is based on a data logger of Campbell Scientific mod. CR10 complete with two multiplexers for the extension of acquisition channels of analog data and contact closure. The programmability of the datalogger has allowed to establish the frequency with the maximum freedom for the acquisition and storage of data, allowing it to suspend all operations in the case of power failure. This allows the battery to hold a charge up to 4 days and then to preserve the CR10 from the loss of the program and data, and the system to resume working properly to restore the power supply.

CR10 has a circular buffer data memory, so the data stored once saturated the capacity of the data logger, go to fill the memory locations in which they were already stored previous acquisitions. The program prepared, described in a later paragraph, has allowed the data memory of the CR10 maintained data for the last six days of acquisition. To overcome this limit, the CR10 has been linked with two serial micromodem to a computer on which a program, at predetermined time intervals, automatically transferred to the hard disk data recorded by CR10 after last query, making the storage capacity of the system virtually unlimited. The CR10 has two program tables, which can be assigned different execution intervals. The first table was used for the acquisition of outflow data and the second for the acquisition of environmental parameters and inflows.

The execution interval of the first table was 5 seconds, and the signals from the level sensors and rain gauge of the drain, which through the appropriate calibration scales, were converted respectively in heights and volumes of water; every two minutes were the heights stored snapshots and the total volume passed through the drain.

The second table acquires every 10 seconds signals from environmental sensors and the three rain gauges and converted in the respective physical variables; every 10 minutes the height of rain was totalized and stored and every 30 minutes the average of the data read from environmental sensors has been stored.

\section{Sensors description}

Acquisition system has been linked to magnetic switches of the three rain gauges. For the acquisition of meteorological data, the system has been connected to sensors for detecting speed and wind direction, temperature, relative air humidity and solar radiation.

For the measurements of the levels in the tank, were used probes that return a current signal, so that the measures were not influenced by the length of the connecting cables to the datalogger. Initially, they were used to position sensors that exploit the principle of magnetostriction created by a permanent magnet placed in a point of a conductor; in a second time levels were measured with pressure transducers (designed to work in adverse conditions) characterized by a single circuit for the power supply and measurement.

For drain outflow measurement a rain gauge was installed with contact closure device connected with the effluent flow from the drains and the measurement was carried out as is set for the measurement of inflows.

\section{Soil sensors}

For the acquisition of data relating to soil, in particular water content and potential, in two plots were prepared 6 measurement stations equipped with an access tube for the probe to neutrons, three TDR probes (Topp, 1980) and two tensiometers. The aluminium tube for the neutron probe access has been driven into the soil to a depth of $1.5 \mathrm{~m}$; the measurements were made weekly at six different depths, in the range $20-110 \mathrm{~cm}$. The tensiometers were positioned at a depth of $80 \mathrm{~cm}$ and $100 \mathrm{~cm}$. The readings were taken every three to four days. The neutron probe measurements of water content, allow good spatial resolution but, being related to the presence of an operator in the field, have a limited temporal frequency. To overcome this drawback, the stations have been completed with three TDR probes for the automatic measurement at three different depths, more specifically, a probe has been stuck in the surface region in vertical position between $0 \mathrm{~cm}$ and $15 \mathrm{~cm}$, the other two positioned horizontally at depth $30 \mathrm{~cm}$ and $60 \mathrm{~cm}$.

The instrument used for the TDR measurements is Tektronix 1502C equipped with a serial interface (SP 232) that allows the connection to the computer through the RS 232 serial port.

To be able to simultaneously detect soil moisture in several places, a multiplexer was built, controlled through the computer, which allows the connection to the tester up to 36 TDR probes. The multiplexer consists of seven coaxial relays in six positions, characterized by an insertion loss, for signals with a frequency of $18 \mathrm{GHz}$, lower than $0.5 \mathrm{~dB}$. The tester is connected to a relay to the outputs of which were connected inputs of the other six relays. In this way they are made 36 ports for the connection of the probes

The probes used were built with two steel conductors of diameter 0.5 $\mathrm{cm}$, length $15 \mathrm{~cm}$, with a distance of $5 \mathrm{~cm}$ and the balun drowned in the resin that constitutes the head of the probe to minimize the dispersion of the signal (Zegelin et al., 1989). Since the theoretical calculations for the construction of the balun does not produce accurate results in reality (Spaans and Baker, 1993), because of uncertainty relative to the properties of ferrites and the components of the coils, the best way to identify suitable materials is a trial and error procedure (Grant and Phillips, 1990).

The control of the TDR acquisition system was operated by an already developed software (Damiani, 1996) originally developed for the single measurement acquisition, modified using the libraries provided in Quick Basic to control the multiplexer (Heimovaara and Bouten, 1990). 


\section{Results}

In the graph of Figure 3 shows the time course of water content, at all three measuring depth, measured by the neutron probe and TDR, as well as the rainfall occurred during the observation period from March 1997 to December 1997. The relative homogeneity found between the measurements carried out in six different locations of the same parcel has permitted the use of average values of the observed quantities. During periods in which it was possible to carry out measurements using both the neutron probes and the TDR, the comparison verifies a substantial agreement in the time evolution of the medium-term water content. As expected on the other hand for the considerations previously developed, the reflectometric method allows to follow this trend with greater detail, highlighting all the short-term variations in the water content in the three horizons also in response to modest rainfall. In contrast, the neutron method, not allowing acquisitions in automatic, is capable of providing only information about the evolutionary trend of the profiles of water content after each rain event.

It is possible to observe how in the initial part of the observation period and following the winter precipitation, the water content in the upper layer reaches values close to saturation and also it is highlight an extremely sensible response to the meteoric inputs. By contrast, the deeper layer shows lower water contents and is less affected and with delay respect the same rain inputs. With the drying of the soil occurring in the summer period a stable water content of $10-15 \%$ is reached for the three layers, then returned to their initial values, according to an evolution which is obviously linked to precipitation events.

The evolution of the profiles of water content acquired using the neutron probe and, alternatively, of TDR measurements was compared with the result of a simulation conducted by applying the model POLICORO (Santini, 1992), widely verified for different soils and crops. The use of the simulation model is particularly useful in the interpretation of experimental tests, in addition to allowing for a relatively easy assessment of the influence of soil and meteorological parameters on the distribution of water in the system.

In the simulation as input data were used the daily actual evapotranspiration data in $\mathrm{mm} / \mathrm{d}$ determined by applying the Pennman-Monteith equation, as well as the rain in $\mathrm{mm} / \mathrm{d}$. The hydrological behaviour of the soil has been described by solving the equation of Richards, using the hydraulic properties, retention curves and hydraulic conductivity (Figure 1). With reference to boundary conditions adopted for the simulation, the surface processes of infiltration and evaporation were controlled by external environmental conditions. In this hypothesis, the evaporation is determined by radiation external conditions until reaching a critical potential to the soil surface; achieved that value instead of the evaporation is governed by the profile of water content in the soil. On this basis the model returns in output, among other things, the actual evapotranspiration as permitted by the actual conditions of soil moisture. At the lower boundary is hypothesized a condition of free drainage. In the input data runoff has been neglected, because in the observation period were absent. As an example, in Figure 4 is represented the comparison between the water content measured by the neutron method and simulated, which refers to the three measuring depth.

The reliability of the experimental methodology adopted is confirmed by the good correspondence between field measurements and simulation results, the latter regardless of the characterizations obtained using laboratory on soil undisturbed samples. Of course, with reference to the TDR measurements, not shown here, the periodic fluctuations are followed by the simulation described in more detail.

The importance of monitoring water content profiles with high time frequency is evident when one wants to follow the evolution of the vol-

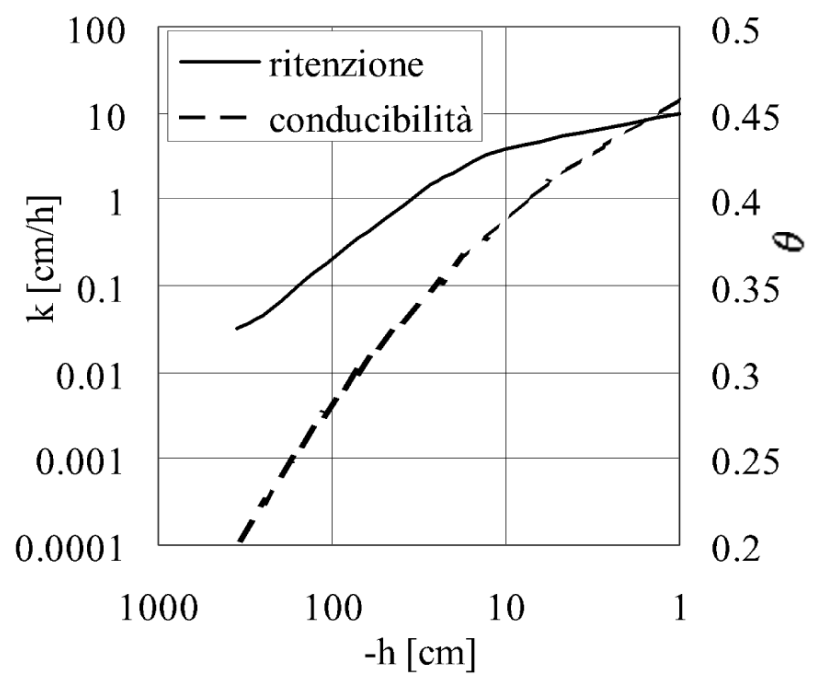

Figure 1. Mean soil hydraulic characteristics.

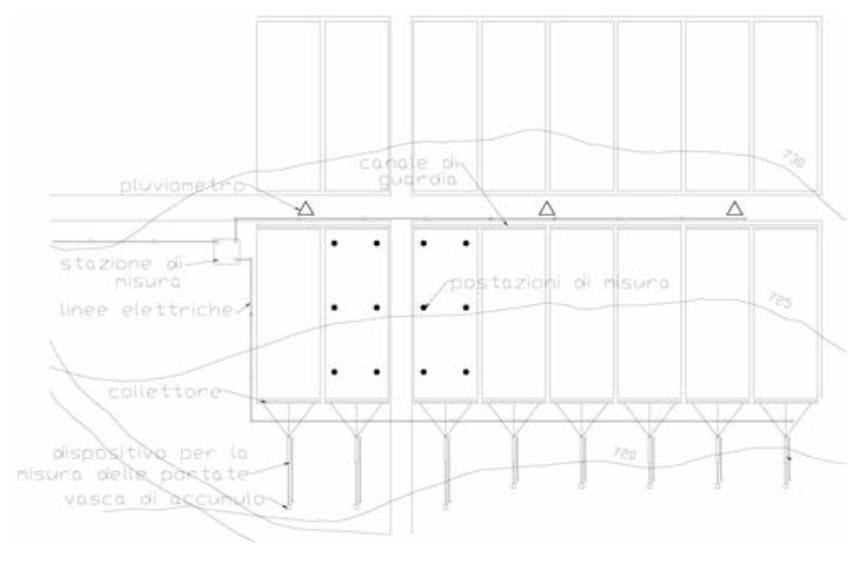

Figure 2. Guardia Perticara experimental plots.

contenuto d'acqua e precipitazioni

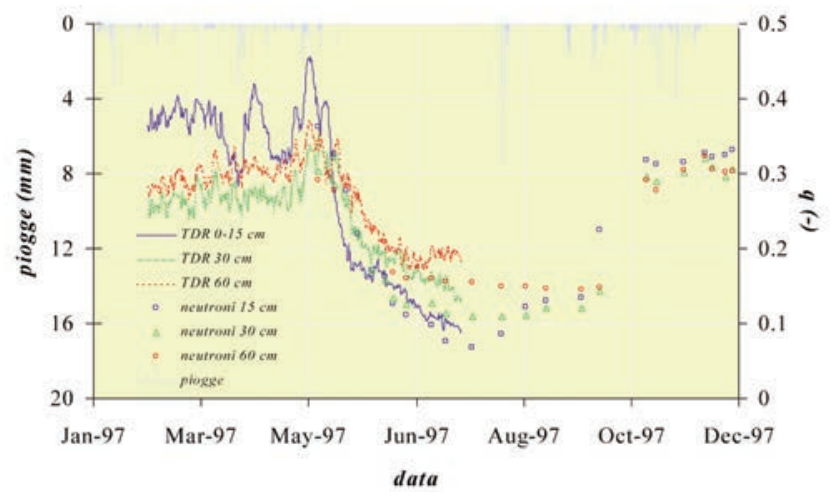

Figure 3. Water content measurements. 

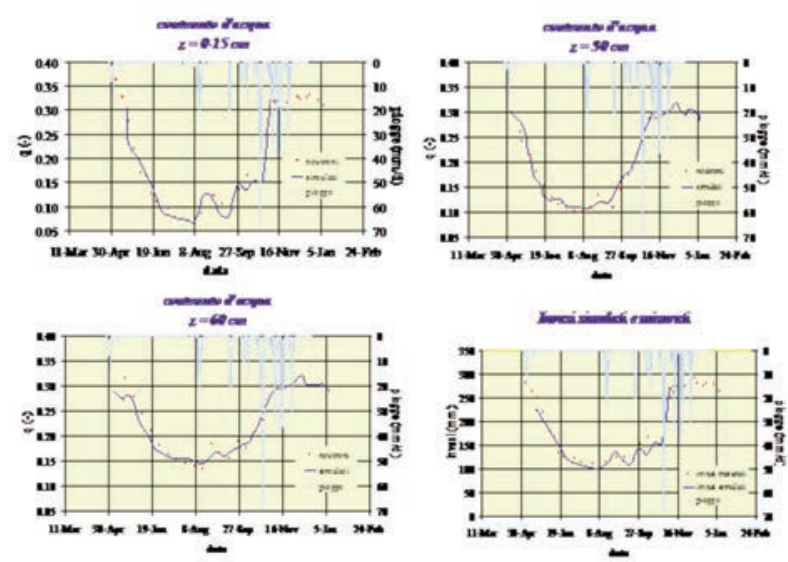

Figure 4. Comparison between experimental and simulated data.

ume of water stored in the soil profile investigated after dry and rainy periods. The fourth graph of Figure 4 shows the trend of the heights of water stored up to a depth of $80 \mathrm{~cm}$, calculated with reference to the profiles of water content measured and simulated respectively. It is a soil that has an average water capacity corresponding to about $170 \mathrm{~mm}$ of water with a maximum in the rainy period of about $280 \mathrm{~mm}$ and a minimum in the dry months of about $110 \mathrm{~mm}$. These values are consistent with those expected given the retention characteristics of the medium under consideration.

\section{Conclusions}

In hydrological sciences is widespread the use of simulation models in the interpretation of real world phenomena. The physical concepts on which the models are often based specifications need to be tested through field experiments that must necessarily involve multi-disciplinary skills. In this sense, the use of sophisticated equipment for the measurement of all variables needed for the quantification of a soil water balance, allowed to describe to a high degree of detail the evolution of the complex exchange processes in the system.

Particular attention was paid to the acquisition and subsequent processing of meteorological and soil data, using instruments that are suitable to be automated, allowing measurements with high spatial and temporal resolution. The reliability of TDR has been verified through the comparison with the neutron method, practically considered a reference for soil water content methods.

The simulation results allow, on the one hand, to verify the usefulness of models in the interpretation of experimental tests, and confirm on the other hand that field experiments conducted through appropriate methodologies are essential for the validation of the models themselves.

\section{References}

Damiani, P., 1996 Tecnica di utilizzo del TDR per la misura del contenuto volumetrico d'acqua nel suolo. Dipartimento TecnicoEconomico per la gestione del Teritorio Agricolo-Forestale.

Grant, I.S., Philips, W.R., 1990: Electromagnetism. John Wiley \& Sons, Chichester, England.

Heimovaara, T.J., Bouten, W., 1993: A computer-controlled 36 channel time domain reflectometry systemfor monitoring soil water contents. Water Resour. Res. 26, 2311-2316.

Hillel, D., 1980: Applications of soil physics. Academic press.

Santini, A., 1992: Modelling water dynamics in the soil-plant-atmosphere system for irrigation problems. Excerpta 6, 133-166.

Santini, A., Romano, N., Ciollaro, G., Comegna, V., 1995: Evaluation of a laboratory inverse method for determining unsaturated hydraulic properties of a soil under different tillage practices. Soil Sci. 160 (5),340-351.

Simunek, J., Sejna, M., Van Genuchten, M. Th., 1998: The HYDRUS-1D software package for simulating water flow and solute transport in two dimensional variably saturated media. International Grownd Water Modeling Center, Colorado

Sommella, A., Gaetani, G., 1990: Impianto sperimentale per la misura del deflusso superficiale e dell'erosione. Pubblicazione dell'Università degli Studi della Basilicata. Istituto di Genio Rurale e Forestale.

Spaans, E.J.A., Baker, J.M., 1993: Simple baluns in parallel probes for Time Domain Reflectometry. Soil Sci. Soc. Am. J. 57, 668-673.

Topp, G.C., 1980: Electromagnetic determination of soil water content: measurements in coaxial trasmission lines. Water Resour. Res. 16, 574-582.

Zegelin, S.J., White, I., Jenkins, D.R., 1989: Improved fields probes for soil water content and electrical conductivity measurements using time domain reflectomety. Water Resour. Res. 25, 2367-2376. 